\title{
Insulin-like growth factor binding protein-3 (IGFBP-3) genetic variant and the risk of esophageal squamous cell carcinoma in a Chinese population
}

H.-P. Yang ${ }^{1}$, J.-F. Liu ${ }^{1}$, J. Rao ${ }^{2}$, X.-M. Zhang ${ }^{2}$, H.-L. Qian ${ }^{3}$, X.-Q. Niu ${ }^{4}$ and Z.-L. Zhao ${ }^{5}$

${ }^{1}$ Department of Thoracic Surgery, Fourth Hospital, Hebei Medical University, Shijiazhuang, Hebei Province, China

${ }^{2}$ Institute of Molecular Genetics, Hebei United University, Tangshang, Hebei Province, China

${ }^{3}$ State Key Laboratory of Molecular Oncology, Cancer Hospital/Institute, Chinese Academy of Medical Sciences, Beijing, China

${ }^{4}$ Department of Thoracic Surgery, Cixian People's Hospital, Handan, Hebei Province, China

${ }^{5}$ Department of Cardiothoracic, Zhongshan Hospital, Dalian University,

Dalian, Liaoning Province, China

Corresponding author: J.-F. Liu

E-mail: junfengliucn@163.com

Genet. Mol. Res. 13 (2): 4146-4153 (2014)

Received September 30, 2013

Accepted February 10, 2014

Published May 30, 2014

DOI http://dx.doi.org/10.4238/2014.May.30.10

\begin{abstract}
Insulin-like growth factor binding protein-3 (IGFBP-3) exerts anti-proliferative or pro-apoptotic effects through IGF-dependent as well as IGF-independent mechanisms in vitro. The purpose of this study was to examine the association between genetic variants in IGFBP-3 (rs2270628) and the risk of esophageal squamous cell carcinoma (ESCC) in a Chinese Han population. Five hundred ESCC cases and 500 cancer-free controls of the Chinese Han population were
\end{abstract}


involved in this study. The IGFBP-3 single-nucleotide polymorphism (SNP) rs2270628 was genotyped and the estimated adjusted odds ratios (ORs) and 95\% confidence intervals (CIs) for its association with the risk of ESCC were determined using unconditional logistic regression analysis. Compared with the rs 2270628 CC genotype, TT genotype was associated with a significantly increased ESCC risk with OR $(95 \% \mathrm{CI})$ of 2.07 (1.05-4.09), but CT genotype was not (OR $=1.25,95 \% \mathrm{CI}$ $=0.94-1.66)$. IGFBP-3 SNP rs2270628 may contribute to the risk of ESCC in the Chinese Han population.

Key words: Esophageal squamous cell carcinoma; IGFBP-3; Genetic polymorphism; Molecular epidemiology

\section{INTRODUCTION}

Esophageal cancer, an aggressive carcinoma, is the 6th most common cause of cancerrelated deaths worldwide (Jemal et al., 2011); it was the 4th leading cause of cancer deaths and the 5th most commonly diagnosed cancer in China in 2009 (Chen et al., 2013). Esophageal cancer includes 2 major histological types: esophageal squamous cell carcinoma (ESCC) and esophageal adenocarcinoma (EAC). ESCC is the predominant histological type of esophageal cancer in China. The major risk factors for ESCC are not well understood, but are thought to include poor nutritional status, low intake of fruit and vegetables, and drinking beverages at high temperatures (Islami et al., 2009a,b; Wu et al., 2009). Although the environment and exposure are both important factors in the development of esophageal carcinogenesis, genetic factors also play an important role (Hiyama et al., 2007). Previous studies have suggested that genetic polymorphisms contribute to the mechanisms involved in esophageal carcinogenesis (Xing et al., 2003). A growing number of genes implicated in alcohol metabolism, folate metabolism, carcinogen metabolism, DNA repair, and cell cycle regulation may be associated with esophageal carcinogenesis (Hiyama et al., 2007).

The insulin-like growth factor (IGF) system is composed of a family of interacting ligands, receptors, and IGF-binding proteins (IGFBPs). IGF-I is a mitogen that plays a pivotal role in regulating cell proliferation, differentiation, and apoptosis (Khandwala et al., 2000). The major binding protein of IGF-I, IGFBP-3, exerts anti-proliferative or proapoptotic effects through IGF-dependent as well as IGF-independent mechanisms in vitro (Baxter, 2000, 2001; Firth and Baxter, 2002). Epidemiological studies have indicated that high levels of IGF-I and low levels of IGFBP-3 are associated with an increased risk of several common cancers, including prostate, breast, lung, and colorectal cancers (Yu and Rohan, 2000; Renehan et al., 2004; Gallagher and LeRoith, 2010). Results regarding the association between genetic variants of $I G F B P-3$ and common cancers have been inconsistent. A previous study reported that IGFBP-3 expression was inversely correlated with the clinical pathological classification of ESCC (Zhao et al., 2012). Various researchers have studied the association between genetic polymorphisms of IGFBP-3 and common cancers, one of which, the rs2270628 single-nucleotide polymorphism (SNP) of IGFBP-3, was associated with an increased risk of ovarian cancer (Terry et al., 2009; Pearce et al., 2011), but was not associated with EAC (McElholm et al., 2010). 
The association between genetic polymorphisms of IGFBP-3 and the risk of ESCC has not been evaluated in previous studies. Therefore, we investigated the association between the IGFBP-3 rs2270628 SNP and the risk of ESCC in a hospital-based case-control study in a Chinese Han population.

\section{MATERIAL AND METHODS}

\section{Study population}

Eligible ESCC cases in this study were consecutively recruited between December 2010 and July 2012 from LuHe Hospital, Tangshan People's Hospital and Cixian People's Hospital, China. Once ESCC cases were diagnosed using histopathology methods, they were immediately contacted by the interviewer at the hospital. All patients in the study were of Chinese Han ethnicity, had lived locally for at least 5 years, were newly diagnosed with ESCC, and were in a stable medical condition as determined by their physician. A total of 500 ESCC cases consented to participate in the study and provide blood samples, giving a response rate of 93.5\% (500/535). Eligible controls included Chinese Han in-patients and cancer-free individuals who had lived in the area for at least 5 years and reported no history of cancer. Controls were matched to the cases according to age at the time of enrollment (within 5 years) and gender. A total of 580 potential cancer-free controls were approached and $500(86.2 \%)$ completed the interview and donated blood samples.

A standard epidemiologic questionnaire was used to determine demographic and lifestyle characteristics, such as gender, age, tobacco smoking, and alcohol consumption. Individuals who smoked once per day for over 1 year were defined as smokers, and smoking history was recoded as never or ever. Individuals who consumed 3 or more alcoholic drinks per week for over 6 months were considered to be alcohol drinkers. After the interview, a 5-mL venous blood sample was collected from each participant. This study was conducted in accordance with the Declaration of Helsinki. This study was conducted with approval from the Ethics Committee of Hebei Medical University. Written informed consent was obtained from all participants.

\section{Genotyping}

Genomic DNA was extracted from the buffy-coat fraction of the blood samples using the DP318-08 Blood DNA Mini Kit (Tiangen Biotech Co. Ltd.; Beijing, China). The rs2270628 SNP was genotyped using the TaqMan real-time polymerase chain reaction (PCR) method with a 7900 HT Fast Real-time PCR system (Applied Biosystems; Foster City, CA, USA). The primers for amplifying the IGFBP-3 fragment were 5'-TCA CTG TGG AAA TTG AGA TTG T-3' (sense) and 5'-AGA AAA GCA GAA TGC AAT TAT ATT GTG-3' (antisense). PCR was accomplished using $2.5 \mu \mathrm{L}$ Premix ExTaq (Jikang Biotechnology Co., Ltd.; Shanghai, China), with $0.125 \mu \mathrm{L}$ sense and antisense primers and $0.2 \mu \mathrm{L}$ probe. Reaction conditions involved an initial denaturation step for $2 \mathrm{~min}$ at $50^{\circ} \mathrm{C}$, followed by 42 cycles at $95^{\circ} \mathrm{C}$ for 10 min, $95^{\circ} \mathrm{C}$ for $15 \mathrm{~s}$, and $60^{\circ} \mathrm{C}$ for $1 \mathrm{~min}$. Endpoint analysis was conducted using the Allelic Discrimination Program of the SDS Image Analysis Software (Version 2.4). To ensure high genotyping accuracy, strict quality control procedures were implemented. Approximately $5 \%$ of samples underwent repeated genotyping, and the results were concordant. 


\section{Statistical analysis}

Differences between ESCC cases and control subjects in the frequency distributions of the selected demographic variables, risk factors, and each of the alleles and genotypes of the SNP were evaluated using the $\chi^{2}$ test. Unconditional logistic regression analyses were used to calculate odds ratios (ORs) to estimate the risk of ESCC and $95 \%$ confidence intervals ( $95 \% \mathrm{CIs}$ ). Multivariate adjustments were made when appropriate for age and gender in further stratification analysis of genotype data. The Hardy-Weinberg equilibrium for genotype distribution in controls was tested using a goodness-of-fit $\chi^{2}$ test. Statistical significance was established as $\mathrm{P}<0.05$, and all tests were 2-sided and performed using SPSS 16.0 (SPSS; Chicago, IL, USA).

\section{RESULTS}

\section{Population characteristics}

Characteristics of the 500 confirmed ESCC cases and 500 cancer-free controls are summarized in Table 1 . Approximately $80 \%$ of subjects in each group were male. The mean (standard deviation) age was $58.3 \pm 8.6$ years in patients, and $59.6 \pm 8.5$ years in controls. There were no significant differences in age and gender between the cases and controls $(\mathrm{P}=$ 0.96 and $\mathrm{P}=0.527$, respectively). However, ESCC cases were more likely to be smokers (61.2 vs $29.2 \%, \mathrm{P}<0.001)$ or drinkers $(26.2$ vs $15.4 \%, \mathrm{P}<0.001)$ than the controls. Among smokers, there was no significant difference between groups in the number of cigarettes smoked.

\begin{tabular}{|c|c|c|c|}
\hline & \multicolumn{2}{|c|}{ Group } & \multirow[t]{2}{*}{$P$ value } \\
\hline & Case $(\mathrm{N}=500)$ & Control $(\mathrm{N}=500)$ & \\
\hline Gender (N, \%) & & & 0.527 \\
\hline Male & $404(80.8)$ & $396(79.2)$ & \\
\hline Female & $96(19.2)$ & $104(20.8)$ & \\
\hline Age (years) (N, \%) & & & 0.96 \\
\hline$<50$ & $74(14.8)$ & $70(14.0)$ & \\
\hline$\sim 50$ & $170(34.0)$ & $174(34.8)$ & \\
\hline$\sim 60$ & $172(34.4)$ & 168 (33.6) & \\
\hline$\sim 70$ & $84(16.8)$ & $88(17.6)$ & \\
\hline Smoking status (N, \%) & & & $<0.001$ \\
\hline Non-smoker & $194(38.8)$ & $354(70.8)$ & \\
\hline Smoker & $306(61.2)$ & $146(29.2)$ & \\
\hline Pack-years smoked (N, \%) & & & 0.065 \\
\hline$<30$ & $137(44.8)$ & $52(35.6)$ & \\
\hline$\geq 30$ & $169(55.2)$ & $94(64.4)$ & \\
\hline Alcohol drinking (N, \%) & & & $<0.001$ \\
\hline Non alcohol drinker & $369(73.8)$ & $423(84.6)$ & \\
\hline Alcohol drinker & $131(26.2)$ & 77 (15.4) & \\
\hline
\end{tabular}

2-sided $\chi^{2}$ test.

\section{IGFBP-3 genotype distributions and association with ESCC risk}

Genotype distributions of IGFBP-3 rs 2270628 in the cases and controls are shown in Table 2. Observed genotype frequencies for the polymorphisms were in Hardy-Weinberg equilibrium in the control group $(\mathrm{P}=0.77)$. The genotype frequencies were $64.2 \% \mathrm{CC}, 30.6 \%$ 
$\mathrm{CT}$, and $5.2 \%$ TT in cancer cases and 70.4\% CC, $26.8 \% \mathrm{CT}$, and $2.8 \% \mathrm{TT}$ in controls. Logistic regression analyses further revealed that TT homozygotes showed a significant 2.07 -fold increased risk of $\mathrm{ESCC}(95 \% \mathrm{CI}=1.05-4.09, \mathrm{P}=0.035)$.

$\begin{aligned} & \text { Table 2. Genotype frequencies of } I G F B P-3 \text { among cases and controls and their association with esophageal } \\
& \text { cancers. }\end{aligned}$
\begin{tabular}{lcccc}
\hline$I G F B P-3$ & Control $(\mathrm{N}=500)$ & Case $(\mathrm{N}=500)$ & OR $(95 \% \mathrm{CI})^{\mathrm{a}}$ & P value \\
\cline { 2 - 4 } genotype & $\mathrm{N}(\%)$ & $\mathrm{N}(\%)$ & & \\
\hline CC & $352(70.4)$ & $321(64.2)$ & & 0.120 \\
CT & $134(26.8)$ & $153(30.6)$ & $1.25(0.94-1.66)$ & 0.035 \\
\hline TT & $14(2.8)$ & $26(5.2)$ & $2.07(1.05-4.09)$ & \\
\hline
\end{tabular}

$\mathrm{OR}=$ odds ratio; $95 \% \mathrm{CI}=95 \%$ confidence interval. ${ }^{a}$ Data were calculated by unconditional logistic regression, with the $\mathrm{CC}$ genotype as the reference group and adjusted for gender, age, and smoking and drinking status.

\section{Association of ESCC risk and IGFBP-3 polymorphisms by smoking status}

Stratification analysis was conducted to evaluate the association between the IGFBP-3 SNP rs 22706228 and ESCC risk by smoking status. As shown in Table 3, compared with carriers of the CC genotype, those with the CT or TT genotypes showed a $37 \%$ increased risk in nonsmokers $(\mathrm{OR}=1.37,95 \% \mathrm{CI}=1.01-2.00, \mathrm{P}<0.001)$. Carriers of the $\mathrm{CT}$ or TT genotypes showed a significant 5.93-fold increased risk in smokers $(\mathrm{OR}=5.93,95 \% \mathrm{CI}=3.78-9.31, \mathrm{P}<0.001)$.

Table 3. Risk of esophageal cancer association with $I G F B P-3$ genotype by smoking status.

\begin{tabular}{lcccc}
\hline Smoking status & \multicolumn{4}{c}{ IGFBP-3 genotype } \\
\cline { 2 - 5 } & $\mathrm{CC}^{\mathrm{a}}$ & OR $(95 \% \mathrm{CI})^{\mathrm{b}}$ & $\mathrm{CT}^{\mathrm{b}}+\mathrm{TT}^{\mathrm{a}}$ & OR $(95 \% \mathrm{CI})^{\mathrm{b}}$ \\
\hline Non-smoker & $124 / 247$ & $1.00($ reference $)$ & $70 / 107$ & $1.37(1.01-2.00)$ \\
Smoker & $197 / 105$ & $4.16(2.90-5.96)$ & $109 / 41$ & $5.93(3.78-9.31)$ \\
$<30$ pack-years & $92 / 35$ & $5.82(3.62-9.34)$ & $45 / 17$ & $5.61(3.00-10.45)$ \\
$\geq 30$ pack-years & $105 / 70$ & $3.11(2.07-4.69)$ & $64 / 24$ & $1.88(1.07-3.59)$ \\
\hline
\end{tabular}

$\mathrm{OR}=$ odds ratio; $95 \% \mathrm{CI}=95 \%$ confidence interval. ${ }^{\mathrm{a} N u m b e r}$ of cases/number of controls. ${ }^{\mathrm{b}} \mathrm{Data}$ were calculated by logistic regression, with the $\mathrm{CC}$ genotype as the reference group and adjusted for age, gender, and drinking status.

\section{Association of ESCC risk and IGFBP-3 polymorphisms by drinking status}

The same stratification analysis was executed to evaluate the association between the IGFBP-3 SNP rs 22706228 and ESCC risk by drinking status. As shown in Table 4, compared with carriers of the CC genotype, carriers of the CT or TT genotypes showed a $39 \%$ increased risk in non-drinkers $(\mathrm{OR}=1.39,95 \% \mathrm{CI}=1.03-1.88, \mathrm{P}<0.05)$. Carriers of the $\mathrm{CT}$ or TT genotypes showed a $99 \%$ increased risk in drinkers $(\mathrm{OR}=1.99,95 \% \mathrm{CI}=1.17-3.40, \mathrm{P}<0.05)$.

Table 4. Risk of esophageal cancer association with IGFBP-3 genotype by drinking status.
\begin{tabular}{lcccc}
\hline Drinking status & \multicolumn{3}{c}{ IGFBP-3 genotype } \\
\cline { 2 - 5 } & $\mathrm{CC}^{\mathrm{a}}$ & $\mathrm{OR}(95 \% \mathrm{CI})^{\mathrm{b}}$ & $\mathrm{CT}^{\mathrm{T}} \mathrm{TT}^{\mathrm{a}}$ & $\mathrm{OR}^{\mathrm{a}}(95 \% \mathrm{CI})^{\mathrm{b}}$ \\
\hline Non-drinker & $234 / 300$ & $1.00($ (reference) & $135 / 123$ & $1.39(1.03-1.88)$ \\
Drinker & $87 / 52$ & $1.80(1.20-2.72)$ & $44 / 25$ & $1.99(1.17-3.40)$ \\
\hline
\end{tabular}

$\mathrm{OR}=$ odds ratio; $95 \% \mathrm{CI}=95 \%$ confidence interval. ${ }^{\mathrm{a}}$ Number of cases/number of controls. ${ }^{\mathrm{b}}$ Data were calculated by logistic regression, with the $\mathrm{CC}$ genotype as the reference group and adjusted for age, gender, and smoking status. 


\section{DISCUSSION}

This is the first study to investigate the association between the IGFBP-3 SNP rs2270628 and risk of ESCC. This hospital-based case-control study in a Chinese Han population indicated that the rs2270628 T allele was associated with a significantly increased risk of ESCC.

IGFBP-3 is located on chromosome $7 \mathrm{p} 13$ and contains 5 exons. The protein binds more than $90 \%$ of circulating IGF-I and forms a ternary complex with an acid-labile subunit. IGFBP-3 regulates normal and malignant cell growth through mechanisms that are dependent or independent of IGF-I binding (Baxter, 2000, 2001; Khandwala et al., 2000; Firth and Baxter, 2002). Previous studies have demonstrated that IGFBP-3 has anti-proliferative and proapoptotic effects, which are mediated through a specific cell surface receptor (Firth and Baxter, 2002). Epidemiological studies have shown that high circulating levels of IGFBP-3 were associated with a low risk of development of several common cancers, including prostate, colorectal, breast, and lung cancers, but these associations were modest and varied between different cancer sites (Renehan et al., 2004). In addition, a study conducted in the US reported that colorectal cancer patients with the IGFBP-3 G2133C variant allele had lower circulating IGFBP-3 levels and an increased risk of colorectal cancer (Le Marchand et al., 2005). Another study reported that the percentage of positive IGFBP-3 staining in ESCC tissues was significantly lower than that in non-malignant tissues; IGFBP-3 expression was inversely correlated with the clinical pathological classification of ESCC, and patients with low levels of IGFBP-3 expression showed reduced overall survival (Zhao et al., 2012). These inverse associations between IGFBP-3 levels and risk of ESCC, breast cancer, and colorectal cancer indicate that IGFBP-3 plays a protective role against cancer.

In a twin study, both genetic and lifestyle factors affected the levels of IGF-I and IGFBP-3, while genetic variation accounted for $60 \%$ of IGFBP-3 levels in serum (Harrela et al., 1996; Holmes et al., 2002); however, the specific loci involved were not determined. Polymorphisms that alter gene expression or protein function may result in a relative increase or decrease in circulating IGFBP-3 levels and may influence carcinogenesis. Recent studies have examined IGFBP-3 SNPs, but the number of studies on SNP rs2270628 is very limited. $\mathrm{Su}$ et al. (2010) found that circulating IGF-I levels were not associated with proliferative benign breast disease risk, although the minor allele of the IGFBP-3 SNP rs2270628 was associated with higher IGF-I levels. In a cross-sectional study of 1121 control subjects, Tamimi et al. (2007) concluded that none of the genetic variations within IGFBP-3 were significantly associated with mammographic density. The Breast and Prostate Cohort Consortium genotyped IGF-I, IGFBP-1, and IGFBP-3 SNPs in 6912 breast cancer cases and 8819 matched controls and observed no statistical associations between rs2270628 and prostate cancer risk (Gu et al., 2010; Schumacher et al., 2010; Tsilidis et al., 2013). In a pooled analysis, Terry et al. (2009) identified that IGFBP-3 SNP rs2270628 was associated with IGF-I plasma levels as well as with an increased risk of ovarian cancer in UK and New England (USA) populations. Similar results were reported in other studies (Pearce et al., 2011; Malaguarnera et al., 2012). No association was observed between rs2270628 and endometrial cancer risk (McGrath et al., 2011). A population-based study demonstrated that the IGFBP-3 SNP rs2270628 was not associated with EAC (McElholm et al., 2010). However, in the current study in a Chinese Han population, we found that rs2270628 was associated with a significantly increased risk of ESCC. The discrepancies in the results may be related to different hereditary backgrounds and 
etiologies. For example, the frequency of the rs2270628 T allele in the Chinese Han population is 0.15 , but is 0.19 in Caucasians (National Center for Biotechnology Information; http:// www.ncbi.nlm.nih.gov). Another possibility may be related to the different etiologies of the primary tumors, because the epidemiology and pathogenesis of ESCC and EAC are different (Anonymous, 2008).

Although our finding of an association between IGFBP-3 rs2270628 and ESCC is novel, the evidence is preliminary and further studies are needed to confirm our results. Interestingly, compared with subjects with the CC genotype, those with the T allele showed a significantly increased risk of ESCC in non-smokers and smokers. This may be because cigarette smoking is more of a promoter of carcinogenesis than a mutagenic initiator (Sharp et al., 2001; Znaor et al., 2003; Castellsagué et al., 2004; Freedman et al., 2007; Holmes and Vaughan, 2007; Ishiguro et al., 2009; Wu et al., 2006; 2009). Similarly, carriers of the T allele had a significantly increased risk of ESCC in non-drinkers and drinkers compared with subjects with the CC genotype, indicating that alcohol metabolism may be associated with esophageal carcinogenesis (Hiyama et al., 2007).

Limitations of this study include the following. First, the sample size of this study was small. Second, the results were not adjusted for other probable risk factors for ESCC (e.g., dietary habits and lifestyle factors) because this information was not available. Third, because the control group was from a hospital cohort, it may not reflect the true genotype distribution of the Chinese Han population. In addition, our study lacked information on the phenotype of serum levels of IGFBP-3, limiting further analysis of the genotype-phenotype correlation.

In conclusion, our study demonstrated that IGFBP-3 SNP rs2270628 may contribute to the risk of ESCC in the Chinese Han population.

\section{ACKNOWLEDGMENTS}

The authors are grateful to the staff of the Institute of Molecular Genetics of Hebei United University. Furthermore, we thank all physicians involved in this study for help with data management and blood sampling. Haiping Yang works in LuHe Hospital.

\section{REFERENCES}

Anonymous (2008). Esophageal cancer: epidemiology, pathogenesis and prevention. Nat. Clin. Pract. Gastroenterol. Hepatol. 5: 517-526.

Baxter RC (2000). Insulin-like growth factor (IGF)-binding proteins: interactions with IGFs and intrinsic bioactivities. Am. J. Physiol. Endocrinol. Metab. 278: E967-E976.

Baxter RC (2001). Signalling pathways involved in antiproliferative effects of IGFBP-3: a review. Mol. Pathol. 54: 145-148.

Castellsagué X, Quintana MJ, Martínez MC, Nieto A, et al. (2004). The role of type of tobacco and type of alcoholic beverage in oral carcinogenesis. Int. J. Cancer 108: 741-749.

Chen W, He Y, Zheng R, Zhang S, et al. (2013). Esophageal cancer incidence and mortality in China, 2009. J. Thorac. Dis. 5: 19-26.

Firth SM and Baxter RC (2002). Cellular actions of the insulin-like growth factor binding proteins. Endocr. Rev. 23: 824-854.

Freedman ND, Abnet CC, Leitzmann MF, Mouw T, et al. (2007). A prospective study of tobacco, alcohol, and the risk of esophageal and gastric cancer subtypes. Am. J. Epidemiol. 165: 1424-1433.

Gallagher EJ and LeRoith D (2010). The proliferating role of insulin and insulin-like growth factors in cancer. Trends Endocrinol. Metab. 21: 610-618.

Gu F, Schumacher FR, Canzian F, Allen NE, et al. (2010). Eighteen insulin-like growth factor pathway genes, circulating levels of IGF-I and its binding protein, and risk of prostate and breast cancer. Cancer Epidemiol. Biomarkers Prev. 
19: $2877-2887$

Harrela M, Koistinen H, Kaprio J, Lehtovirta M, et al. (1996). Genetic and environmental components of interindividual variation in circulating levels of IGF-I, IGF-II, IGFBP-1, and IGFBP-3. J. Clin. Invest. 98: 2612-2615.

Hiyama T, Yoshihara M, Tanaka S and Chayama K (2007). Genetic polymorphisms and esophageal cancer risk. Int. J. Cancer 121: 1643-1658.

Holmes RS and Vaughan TL (2007). Epidemiology and pathogenesis of esophageal cancer. Semin. Radiat. Oncol. 17: 2-9.

Holmes MD, Pollak MN and Hankinson SE (2002). Lifestyle correlates of plasma insulin-like growth factor I and insulinlike growth factor binding protein 3 concentrations. Cancer Epidemiol. Biomarkers Prev. 11: 862-867.

Ishiguro S, Sasazuki S, Inoue M, Kurahashi N, et al. (2009). Effect of alcohol consumption, cigarette smoking and flushing response on esophageal cancer risk: a population-based cohort study (JPHC study). Cancer Lett. 275: 240-246.

Islami F, Boffetta P, Ren JS, Pedoeim L, et al. (2009a). High-temperature beverages and foods and esophageal cancer risk - a systematic review. Int. J. Cancer 125: 491-524.

Islami F, Pourshams A, Nasrollahzadeh D, Kamangar F, et al. (2009b). Tea drinking habits and oesophageal cancer in a high risk area in northern Iran: population based case-control study. BMJ 338: b929.

Jemal A, Bray F, Center MM, Ferlay J, et al. (2011). Global cancer statistics. CA Cancer J. Clin. 61: 69-90.

Khandwala HM, McCutcheon IE, Flyvbjerg A and Friend KE (2000). The effects of insulin-like growth factors on tumorigenesis and neoplastic growth. Endocr. Rev. 21: 215-244.

Le Marchand L, Kolonel LN, Henderson BE and Wilkens LR (2005). Association of an exon 1 polymorphism in the IGFBP3 gene with circulating IGFBP-3 levels and colorectal cancer risk: the multiethnic cohort study. Cancer Epidemiol. Biomarkers Prev. 14: 1319-1321.

Malaguarnera R, Morcavallo A and Belfiore A (2012). The insulin and igf-I pathway in endocrine glands carcinogenesis. J. Oncol. 2012: 635614 .

McElholm AR, McKnight AJ, Patterson CC, Johnston BT, et al. (2010). A population-based study of IGF axis polymorphisms and the esophageal inflammation, metaplasia, adenocarcinoma sequence. Gastroenterology 139: 204-212.

McGrath M, Lee IM, Buring J and De Vivo I (2011). Common genetic variation within IGFI, IGFII, IGFBP-1, and IGFBP-3 and endometrial cancer risk. Gynecol. Oncol. 120: 174-178.

Pearce CL, Doherty JA, Van Den Berg DJ, Moysich K, et al. (2011). Genetic variation in insulin-like growth factor 2 may play a role in ovarian cancer risk. Hum. Mol. Genet. 20: 2263-2272.

Renehan AG, Zwahlen M, Minder C, O'Dwyer ST, et al. (2004). Insulin-like growth factor (IGF)-I, IGF binding protein-3, and cancer risk: systematic review and meta-regression analysis. Lancet 363: 1346-1353.

Schumacher FR, Cheng I, Freedman ML, Mucci L, et al. (2010). A comprehensive analysis of common IGF1, IGFBP1 and IGFBP3 genetic variation with prospective IGF-I and IGFBP-3 blood levels and prostate cancer risk among Caucasians. Hum. Mol. Genet. 19: 3089-3101.

Sharp L, Chilvers CE, Cheng KK, McKinney PA, et al. (2001). Risk factors for squamous cell carcinoma of the oesophagus in women: a case-control study. Br. J. Cancer 85: 1667-1670.

Su X, Colditz GA, Willett WC, Collins LC, et al. (2010). Genetic variation and circulating levels of IGF-I and IGFBP-3 in relation to risk of proliferative benign breast disease. Int. J. Cancer 126: 180-190.

Tamimi RM, Cox DG, Kraft P, Pollak MN, et al. (2007). Common genetic variation in IGF1, IGFBP-1, and IGFBP-3 in relation to mammographic density: a cross-sectional study. Breast Cancer Res. 9: R18.

Terry KL, Tworoger SS, Gates MA, Cramer DW, et al. (2009). Common genetic variation in IGF1, IGFBP1 and IGFBP3 and ovarian cancer risk. Carcinogenesis 30: 2042-2046.

Tsilidis KK, Travis RC, Appleby PN, Allen NE, et al. (2013). Insulin-like growth factor pathway genes and blood concentrations, dietary protein and risk of prostate cancer in the NCI Breast and Prostate Cancer Cohort Consortium (BPC3). Int. J. Cancer 133: 495-504.

Wu IC, Lu CY, Kuo FC, Tsai SM, et al. (2006). Interaction between cigarette, alcohol and betel nut use on esophageal cancer risk in Taiwan. Eur. J. Clin. Invest. 36: 236-241.

Wu M, Liu AM, Kampman E, Zhang ZF, et al. (2009). Green tea drinking, high tea temperature and esophageal cancer in highand low-risk areas of Jiangsu Province, China: a population-based case-control study. Int. J. Cancer 124: 1907-1913.

Xing D, Tan W and Lin D (2003). Genetic polymorphisms and susceptibility to esophageal cancer among Chinese population (review). Oncol. Rep. 10: 1615-1623.

$\mathrm{Yu} \mathrm{H}$ and Rohan T (2000). Role of the insulin-like growth factor family in cancer development and progression. J. Natl. Cancer Inst. 92: 1472-1489.

Zhao L, He LR, Zhang R, Cai MY, et al. (2012). Low expression of IGFBP-3 predicts poor prognosis in patients with esophageal squamous cell carcinoma. Med. Oncol. 29: 2669-2676.

Znaor A, Brennan P, Gajalakshmi V, Mathew A, et al. (2003). Independent and combined effects of tobacco smoking, chewing and alcohol drinking on the risk of oral, pharyngeal and esophageal cancers in Indian men. Int. J. Cancer 105: 681-686. 